\title{
The Role of Muscle Activation Pattern and Calcineurin in Acetylcholinesterase Regulation in Rat Skeletal Muscles
}

\author{
Peter Pregelj, ${ }^{1 \star}$ Miha Trinkaus, ${ }^{1 \star}$ Daša Zupan, ${ }^{1}$ Jože J. Trontelj, ${ }^{2}$ and Janez Sketelj ${ }^{1}$ \\ ${ }^{1}$ Institute of Pathophysiology, Faculty of Medicine, University of Ljubljana, and 'Institute of Clinical Neurophysiology, Clinical Center, SI-1000 Ljubljana, \\ Slovenia
}

Acetylcholinesterase (AChE) expression in fast rat muscles is approximately fourfold higher than in slow muscles. We examined whether different muscle activation patterns are responsible for this difference and whether the calcineurin signaling pathway is involved in AChE regulation. The slow soleus and fast extensor digitorum longus (EDL) muscles were directly or indirectly stimulated by a tonic lowfrequency or a phasic high-frequency pattern of electric impulses. The phasic, but not tonic, stimulation increased the AChE mRNA levels in denervated soleus muscles to those in the normal EDL and maintained high levels of AChE mRNA in denervated EDL muscles. Therefore, muscle activation pattern is the predominant regulator of extrajunctional AChE expression in rat muscles. Indirect phasic stimulation of innervated muscles, imposed on their natural pattern of neural activation, did not increase the AChE transcript levels in the soleus, whereas a 30\% reduction was observed in the EDL muscles. A low number of impulses per day is therefore prerequisite for high AChE expression. Treatment by tacrolimus and cyclosporin A, two inhibitors of calcineurin (but not by a related substance rapamycin, which does not inhibit calcineurin), increased the levels of AChE transcripts in the control soleus muscles and in tonically electrically stimulated soleus and EDL muscles, even to reach those in the control EDL muscles. Therefore, tonic muscle activation reduces the extrajunctional levels of AChE transcripts by activating the calcineurin signaling pathway. In denervated soleus and EDL muscles, tacrolimus did not prevent the reduction of AChE mRNA levels, indicating that a calcineurin-independent suppressive mechanism was involved.

Key words: acetylcholinesterase; muscle-nerve interaction; calcineurin; tacrolimus; electrical stimulation; nerve impulse pattern

\section{Introduction}

Slow muscle fibers are activated tonically with low-frequency nerve impulses, whereas the fibers in fast muscles are excited with phasic, short, high-frequency bursts of nerve activity (Navarete and Vrbova, 1983; Hennig and Lømo, 1985). Nerve impulse patterns determine phenotypic properties and gene expression in muscle fibers (Lømo et al., 1974; Termin et al., 1989; Ausoni et al., 1990). Fast and slow rat muscles differ also in regard to acetylcholinesterase (AChE) expression (Dettbarn, 1981; Sketelj et al., 1991). AChE is a key functional constituent of the neuromuscular junction but is expressed also extrajunctionally (Hall, 1973; Sketelj and Brzin, 1985; Sketelj, 1994). In the rat, the extrajunctional activity of AChE is fourfold higher in fast than in slow muscles (Dettbarn, 1981; Črešnar et al., 1994; Michel et al., 1994). Chronic low-frequency electrical stimulation of fast muscles reduced their AChE activity and mRNA levels to those in the slow muscles (Sketelj et al., 1997, 1998). However, it is not known

Received Sept. 25, 2006; revised Dec. 1, 2006; accepted Dec. 19, 2006.

This work was supported by Grants P0-0518-0381 and P3-0171-0381 from the Agency for Research and Development of the Republic of Slovenia. The technical assistance of Boris Pečenko is gratefully acknowledged. We thank Dr. Marjan Mihelin for help with EMG recording.

*P.P. and M.T. contributed equally to this work.

Correspondence should be addressed to Janez Sketelj, Institute of Pathophysiology, Faculty of Medicine, University of Ljubljana, Zaloska 4, SI-1000 Ljubljana, Slovenia. E-mail: janez.sketelj@mf.uni-lj.si.

DOI:10.1523/JNEUROSCI.4182-06.2007

Copyright $\odot 2007$ Society for Neuroscience $\quad$ 0270-6474/07/271106-08\$15.00/0 whether a phasic electrical stimulation pattern can increase $\mathrm{AChE}$ expression in slow muscle fibers to the levels present in fast muscles or maintain high $\mathrm{AChE}$ expression in denervated fast muscle fibers, because slow fibers might be intrinsically different from fast fibers or some factors released from the fast motor nerve might be necessary for high AChE expression. It is also not known whether phasic electrical stimulation of muscles, imposed in addition to their natural activation pattern, is able to further increase AChE expression in fast muscles or enhance it in slow muscles.

Calcineurin, a $\mathrm{Ca}^{2+} /$ calmodulin-activated phosphatase, seems to be involved in the regulation of several fiber typespecific proteins (for review, see Olson and Williams, 2000). Although some results supported other possible mechanisms or argued against this hypothesis (Hughes et al., 1997; Calvo et al., 1999; Delling et al., 2000; Swoap et al., 2000; Spangenburg et al., 2001), more detailed studies corroborated the importance of calcineurin in fiber type specification and explained some previous inconsistencies (Serrano et al., 2001; McCullagh et al., 2004). In cultured developing myotubes, the inhibition of calcineurin by cyclosporin A (CsA) significantly increased AChE mRNA levels, whereas the overexpression of the constitutively active calcineurin reduced AChE mRNA levels (Luo et al., 1999). However, it is not known whether the calcineurin signaling pathway is responsible for the differences in AChE expression between mature fast and slow muscles. 
The aim of the present study was to investigate the importance of muscle activation pattern to AChE expression in muscle fibers and to reveal a possible involvement of the calcineurin signaling in the regulation of AChE expression in mature muscles in vivo. We found that (1) different muscle activation patterns are predominantly responsible for the differences in the extrajunctional AChE expression in slow and fast rat muscles, (2) a low number of muscle fiber activations per day is prerequisite for high muscle AChE expression, (3) inhibition of calcineurin in tonically activated muscles stimulates AChE expression, and (4) the reduction of AChE expression after muscle denervation is mediated by another, calcineurin-independent signaling mechanism.

\section{Materials and Methods}

Adult male rats (Wistar strain, 220-300 g) were used in the experiments. Ketalar $(60 \mathrm{mg} / \mathrm{kg}$; Parke-Davis, Wien, Austria) or Bioketan $(60 \mathrm{mg} / \mathrm{kg}$; Vetoquinol Biowet, Gorzow Wielkopolski, Poland) and Rompun ( $8 \mathrm{mg} /$ kg; Bayer, Leverkusen, Germany) were mixed in a syringe and applied intraperitoneally for anesthesia during all surgical procedures. In accordance with national guidelines, the experiments on animals were approved by the Veterinary Administration of the Ministry for Agriculture, Forestry, and Food of Slovenia (permit 323-349/2003-3). All of the animals were maintained on a standard diet with food and water ad libitum, and all efforts were made to minimize the number of animals and their suffering. At the end of the experiment, the rats were killed by exsanguination under anesthesia.

Denervation. The soleus and extensor digitorum longus (EDL) muscles were denervated for $8 \mathrm{~d}$. The sciatic nerve in one leg was exposed and transected in the midthigh region. A piece of the nerve was excised to prevent reinnervation.

Electrical stimulation of the soleus and EDL muscle. The soleus and EDL muscles were electrically stimulated either indirectly via the sciatic nerve or the denervated muscles were stimulated directly. In the first case, stainless-steel electrodes were implanted in the popliteal region on each side of the sciatic nerve in the right hindlimb (Simoneau and Pette, 1988). For direct electrical stimulation of the denervated soleus and EDL muscles, stainless-steel electrodes were implanted on the proximal and distal end of denervated muscles. Denervation of the soleus muscle was achieved by transection of the soleus muscle nerve proper. The proximal nerve stump was deflected back and sutured to the gastrocnemius muscle to prevent reinnervation. The electrode wires were passed subcutaneously to the neck of the animal and sutured to the skin. The external wires, attached to the electrode wires, were protected by a metal casting, which kept them out of the reach of the animal (Ribarič et al., 2000). The external wires were connected through the rotating suspension and contact mechanism to an electrical stimulator producing square wave stimuli. Two patterns of electric stimulation were used: (1) the "tonic" lowfrequency pattern $(10 \mathrm{~Hz}$, biphasic pulse duration of $200 \mathrm{~ms}$, continuous) was applied $16 \mathrm{~h} / \mathrm{d}$ for $8 \mathrm{~d}$, and (2) the "phasic" highfrequency pattern $(150 \mathrm{~Hz}$, train duration of $0.2 \mathrm{~s}$, interval between the trains of $15 \mathrm{~min}$ ) was applied continuously $24 \mathrm{~h}$ for $8 \mathrm{~d}$. The amplitude of stimulation was adjusted daily so that contractions of the stimulated muscle group could be reliably palpated and that stimulation caused no obvious discomfort to the animals. The rats were put in special cages allowing them to move freely without the risk of getting entangled and pulling the electrodes, with the stimulator being $\sim 50 \mathrm{~cm}$ above the rats.

Single-fiber electromyography. The single-fiber electromyography (SFEMG) technique used was essentially the same as that in studies on human patients (Trontelj, 2003). A pair of fine tungsten needles insulated to near the tip were introduced into the proximal one-third of the muscle with tips a few millimeters apart, and a position was found from which it was possible to elicit small twitches in a part of the muscle with rectangular electrical pulses of $<2-5 \mathrm{~mA}$ amplitude and $20 \mu$ s width. Responses of single muscle fibers were recorded with a standard SFEMG electrode, introduced into the twitching area. Stimulus amplitude was adjusted to be well above the threshold for the axonal branch innervating the selected single muscle fiber. Stimulation and recording was performed on a Synergy EMG system (Oxford Instruments Medical, Oxon,
UK). The stimuli were also delivered by the same stimulator and in the identical way as used for phasic nerve stimulation. In some instances, compound responses were also recorded, containing action potentials of probably less than five muscle fibers.

Application of tacrolimus (FK506), CsA, or rapamycin. Tacrolimus (Prograf, Fujisawa, Ireland) was diluted in saline and injected subcutaneously to rats in a dose of $2 \mathrm{mg} / \mathrm{kg}$ body weight once per day for $8 \mathrm{~d}$. CsA (Novartis Pharma, Basel, Switzerland) was applied in a dose of $50 \mathrm{mg} / \mathrm{kg}$ by one daily injection subcutaneously for $8 \mathrm{~d}$. Rapamycin (LC Laboratories, Woburn, MA) was dissolved in $10 \%$ ethanol and $2 \%$ carboxymethylcellulose and injected subcutaneously once a day in a dose of $1.5 \mathrm{mg} / \mathrm{kg}$ for $8 \mathrm{~d}$.

In separate groups of rats, denervation alone or in combination with the application of tacrolimus or CsA was performed. In the control group, the soleus and EDL muscles were denervated for $8 \mathrm{~d}$ as described above. In the second group, $8 \mathrm{~d}$ denervation treatment was combined with application of $2 \mathrm{mg} / \mathrm{kg}$ body weight of tacrolimus or $50 \mathrm{mg} / \mathrm{kg} \mathrm{CsA}$ during the last $5 \mathrm{~d}$ of the denervation period. Contralateral nondenervated muscles served as controls.

RNA isolation, Northern blotting, and quantitative real-time PCR anal$y$ sis. The soleus and EDL muscles were isolated and frozen immediately in liquid nitrogen. In the slow soleus muscles and in the fast sternomastoid (STM) (but not the EDL) muscles, neuromuscular junctions are located in a rather narrow band across the muscle belly. Therefore, in some experiments, a few millimeters broad region of muscle around the intramuscular nerve branches, which can be visualized under the microscope, was excised in the two muscles, so that the extrajunctional regions were studied separately. Control experiments, in which the neuromuscular junctions were visualized by a histochemical reaction for AChE, confirmed that in this way the region containing the junctions could be reliably separated from the extrajunctional regions in these muscles. For Northern blot analysis, samples were pooled from three animals, pulverized in a mortar chilled by liquid nitrogen, and briefly homogenized in a denaturing solution by an Ultra-Turrax homogenizer. Total RNA was isolated, and Northern blot analysis of AChE mRNA levels was performed as described previously (Pregelj et al., 2003). The autoradiograms were quantified densitometrically using an MCID M4 image analyzer (Imaging Research, St. Catharines, Ontario, Canada). The results were normalized to the rRNA level in the sample and expressed as the percentage of the AChE mRNA level in the normal EDL muscle.

For quantitative real-time (RT)-PCR analysis, total RNA was isolated from each isolated muscle with TRIzol reagent (Invitrogen, Carlsbad, CA) according to the instructions of the supplier. For each sample, $1 \mu \mathrm{g}$ of total RNA was reverse transcribed in a $50 \mu \mathrm{l}$ of the reaction mixture using a High Capacity cDNA Archive kit (Applied Biosystems, Foster City, CA). After the initial step at room temperature for $10 \mathrm{~min}$, reverse transcription was performed at $37^{\circ} \mathrm{C}$ for $2 \mathrm{~h}$. Gene-specific primers and probes were available as TaqMan Gene expression assays (Applied Biosystems). Assay Rn00596883_m1 (exon 3-4 boundary) was used for AChE. The 18S rRNA (part number 4319413E) was used as an internal control. For AChE, the minor groove binder probe was labeled with 6-carboxyfluorescein reporter dye, and for $18 \mathrm{~S}$ rRNA the minor groove binder probe was labeled with VIC reporter dye. Quantitative PCR reactions for AChE were performed with undiluted cDNA samples and with 400 times diluted cDNA samples for $18 \mathrm{~S}$ rRNA. The RT-PCR reaction was performed on a 7500 Real-Time PCR System (Applied Biosystems) in $20 \mu$ l of the reaction mixture containing $1 \times$ TaqMan Universal Master Mix (Applied Biosystems), $1 \times$ Gene Expression Assay mix, and $2 \mu \mathrm{l}$ of sample cDNA as a template. Ninety-six-well optical reaction plates, covered by optical adhesive covers (Applied Biosystems), were used. Initial raw data analysis was performed using the Sequence Detection Software version 1.3.1. Relative mRNA levels were calculated by the $\mathrm{ddC}_{\mathrm{T}}$ method, which is described in the Applied Biosystems User Bulletin Number 2 (P/N 4303859). The AChE mRNA levels were normalized to $18 \mathrm{~S}$ rRNA levels.

Statistical analysis. Statistical analysis of the data were performed with a personal computer using the Excel (Microsoft, Seattle, WA) program. The average and SD for each experimental group was calculated, and then the differences between the groups were compared with the Stu- 
dent's $t$ test. The probability level at which the null hypothesis was rejected was 0.05 .

\section{Results}

AChE mRNA levels in muscles, electrically stimulated by different impulse patterns

Previous investigations showed that chronic low-frequency electrical stimulation of the fast EDL muscle, mimicking the activation pattern in the soleus muscle, greatly decreased the levels of AChE activity and mRNA in stimulated EDL muscles (Sketelj et al., 1997, 1998). In the present study, different patterns of electrical stimulation of the denervated soleus muscles were used to study the effect of muscle activation pattern on the AChE mRNA level in the soleus muscle in the absence of innervation. The levels of mRNA encoding the catalytic subunit of AChE in the control and electrically stimulated muscles were analyzed by Northern blots (Fig. 1A). Two AChE transcripts were detected in these muscles as described previously (Črešnar et al., 1994). The intensity of the corresponding two bands on the autoradiograms was analyzed densitometrically, normalized to the rRNA level in the sample, and expressed as the percentage of the AChE mRNA level in the normal EDL muscles (Fig. $1 B$ ). The AChE mRNA levels in the control soleus muscles were $\sim 20-30 \%$ of those in the EDL. A statistically significant decrease of the average AChE mRNA levels was observed in the $8 \mathrm{~d}$ denervated soleus muscles compared with the control soleus muscles $(p<0.05)$. If the denervated soleus muscles were directly electrically stimulated by a chronic, low-frequency tonic impulse pattern, the average AChE mRNA levels in the stimulated muscles did not differ from those in the normal soleus muscles. However, if the denervated soleus muscles had been electrically stimulated for $8 \mathrm{~d}$ by a phasic highfrequency impulse pattern, approximately mimicking the activation pattern of the motor units in the EDL muscle (Hennig and Lømo, 1985), the average levels of AChE mRNA in the stimulated soleus muscles increased approximately fourfold $(p<0.001)$. They reached the AChE mRNA levels in the normal fast EDL muscles. The same high-frequency phasic pattern of electrical stimulation applied to the denervated EDL muscles also essentially prevented a huge decrease of AChE mRNA levels during $5 \mathrm{~d}$ after denervation, keeping it at $\sim 80 \%$ of the control value (Fig. $2 A, B)$.

In the next experiment, the innervated soleus muscles were activated indirectly by electrical stimulation of their motor axons with the same phasic impulse pattern as above, but, because the muscle was innervated, the natural tonic nerve-induced activation pattern of the soleus muscle was preserved in parallel to phasic electrical stimulation that occurred each $15 \mathrm{~min}$. We first checked the ability of the neuromuscular junctions in the soleus muscle to transmit such high-frequency trains of impulses to the muscle fibers. Microelectromyographic registration of the action potentials in the muscle fibers of the soleus muscle, stimulated indirectly through the electrodes sutured close to the sciatic nerve, was performed. Compound action potentials remained of similar amplitude and shape throughout the train, suggesting that there is no drop out of muscle fibers (Fig. 3A). SFEMG recordings of single-fiber responses revealed that they reliably followed stimulation at $150 \mathrm{~Hz}$, without any blocking (Fig. 3B). Even the jitter (response to response latency variation) remained relatively low, suggesting good safety margin of neuromuscular transmission maintained until the end of the train. There was some latency prolongation attributable to slowing of muscle fiber conduction velocity. The same result was obtained also after $6 \mathrm{~d}$ of indirect soleus muscle stimulation through the chronically im-

\section{SOL}
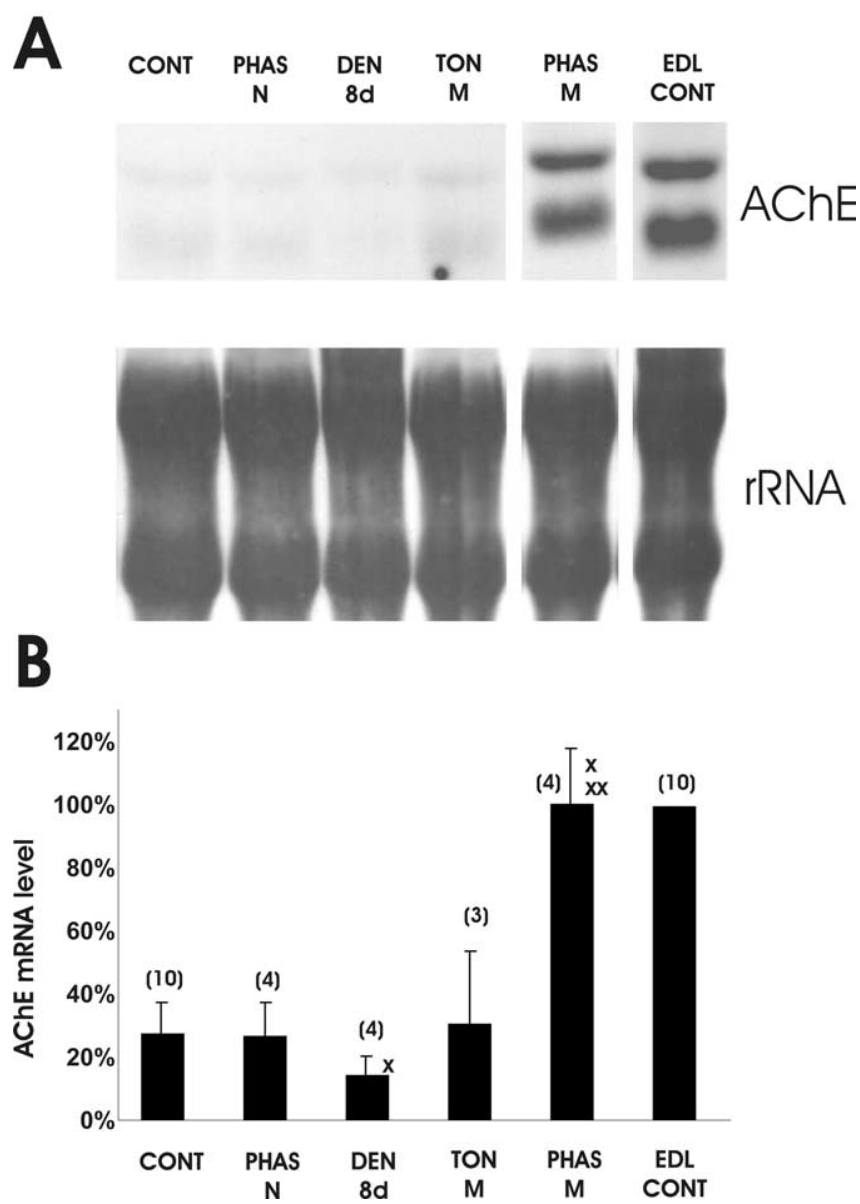

Figure 1. The levels of the AChE mRNA in the soleus (SOL) muscles after $8 \mathrm{~d}$ of phasic or tonic electrical stimulation. $\boldsymbol{A}$, Northern blot analysis. At least three muscles were pooled to obtain the RNA sample for each lane. rRNA staining was used to control the uniformity of RNA loading on the gels. $\boldsymbol{B}$, Quantitative analysis of Northern blots. Muscle AChE mRNA levels were determined densitometrically, normalized to rRNA level in the lane, and expressed as the percentage of the AChE mRNA level in the normal EDL muscles. Mean \pm SD is depicted; the number of experiments is given in parentheses. $x$ indicates statistically significant difference from the control soleus muscle $(p<0.05)$; $x x$ indicates statistically significant difference from the denervated soleus muscle ( $p<0.001$ ). The lanes and columns represent the following muscle samples: CONT, nonstimulated normal muscles of control animals; PHAS N, innervated muscles electrically stimulated for $8 \mathrm{~d}$ via the sciatic nerve by the phasic impulse pattern; DEN 8d, $8 \mathrm{~d}$ denervated nonstimulated muscles; TON M, denervated muscles electrically stimulated by the tonic impulse pattern; PHAS M, denervated muscles electrically stimulated by the phasic impulse pattern.

planted electrodes (figure not shown). These results confirmed that the neuromuscular junctions in the soleus muscle were able to transmit the applied phasic stimulation pattern to the muscle fibers without failure. However, in contrast to directly stimulated soleus muscles, the AChE mRNA levels in the soleus muscles, stimulated via their nerves by the same phasic impulse pattern, did not increase and were not significantly different from those in control muscles $(p>0.05)$ (Fig. $1 A, B)$.

We also examined the effect of the electrical stimulation of the fast EDL muscle via its nerve by the above described phasic highfrequency impulse pattern, imposed on the EDL muscle in addition to its natural neural activation. A statistically significant decrease of the average AChE mRNA levels to $\sim 70 \%$ of the normal value was observed in the $8 \mathrm{~d}$ indirectly phasically stimulated EDL muscles $(p<0.05)$ (Fig. $2 A, B)$. 
EDL

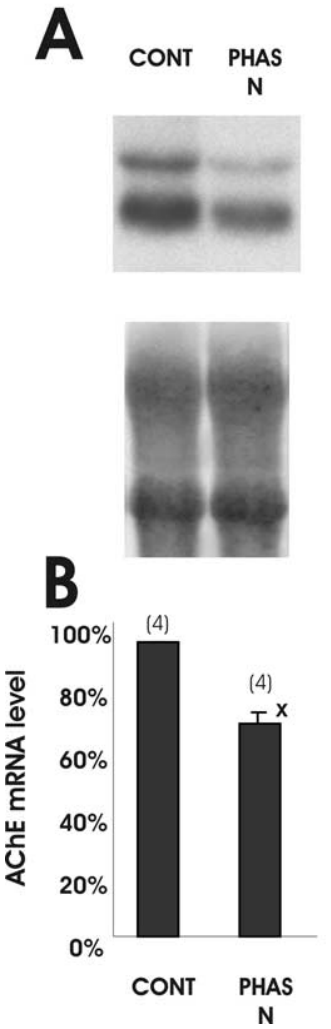

EDL
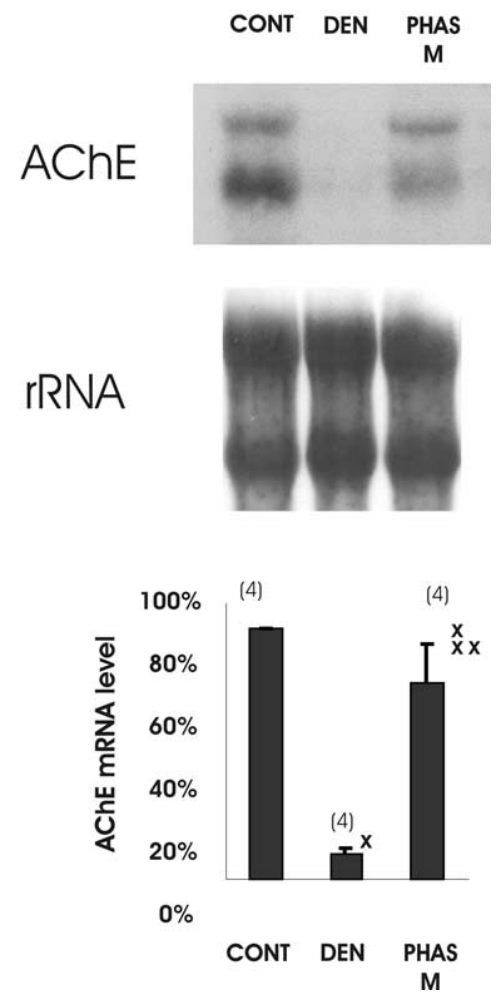

Figure 2. The levels of AChE mRNA in the EDL muscles, electrically stimulated by the phasic impulse pattern. $\boldsymbol{A}$, Northern blot analysis. At least three muscles were pooled to obtain the RNA sample for each lane. rRNA staining was used to control the uniformity of RNA loading on the gels. $\boldsymbol{B}$, Quantitative analysis of Northern blots. Muscle AChE mRNA levels were determined densitometrically, normalized to rRNA level in the lane, and expressed as the percentage of the AChE mRNA level in the normal EDL muscles. Mean \pm SD is depicted; the number of experiments is given in parentheses. The lanes and columns represent the following muscle samples: CONT, nonstimulated normal muscles of control animals; DEN, $5 \mathrm{~d}$ denervated nonstimulated muscles; PHAS M, denervated muscles electrically stimulated for $5 \mathrm{~d}$ by the phasic impulse pattern, starting immediately after denervation; PHAS N, innervated muscles electrically stimulated for $8 \mathrm{~d}$ via the sciatic nerve by the phasic impulse pattern. $\mathrm{x}$ indicates statistically significantly different from CONT ( $p<0.05$ ); xx indicates statistically significantly different from DEN $(p<0.001)$.

\section{AChE expression in the soleus and EDL muscles of the animals treated by calcineurin inhibitors}

In the next group of experiments, rats were treated with two different inhibitors of calcineurin activity to examine a possible involvement of the calcineurin-dependent signaling pathway in muscle AChE regulation. If tacrolimus (FK506) was applied to rats for $8 \mathrm{~d}$, the average AChE mRNA level in the soleus muscles, determined by quantitative RT-PCR, increased approximately fivefold (Fig. 4), the difference was statistically highly significant $(p<0.001)$. This was primarily attributable to the increased extrajunctional expression of AChE because the same phenomenon, as observed in whole muscles, was also observed in the isolated extrajunctional regions of the soleus muscle (see Fig. $6 B$ ).

However, the observed effect of calcineurin inhibitor tacrolimus on the expression of AChE in the soleus muscles might have been attributable to putative changes in motoneuron firing pattern caused by inhibition of calcineurin in motoneurons. To eliminate this potential indirect effect, we examined the consequences of tacrolimus application on the levels of AChE tran-
A

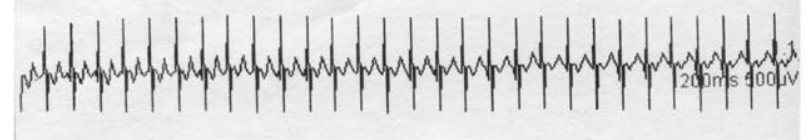

B

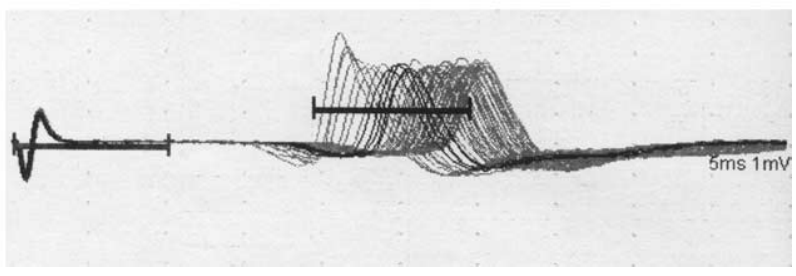

Figure 3. EMG recordings obtained from the soleus muscle, indirectly electrically stimulated by the phasic impulse pattern. $\boldsymbol{A}$, A response to a typical train of impulses $(150 \mathrm{~Hz}$, duration of $0.2 \mathrm{~s}$ ) recorded by a small-needle EMG electrode is depicted. Each stimulation artifact is followed by the compound action potential of a few muscle fibers. $\boldsymbol{B}$, Single-fiber EMG responses to stimulation at $150 \mathrm{~Hz}$. The complete sequence of a $0.2 \mathrm{~s}$ train is shown.

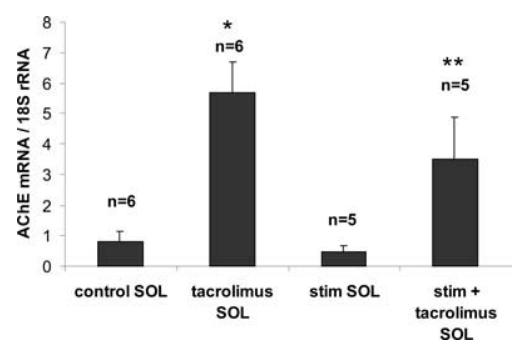

Figure 4. The effect of treatment by tacrolimus (FK506) on the levels of AChE mRNA in the control soleus muscles and in the soleus muscles, electrically stimulated by the tonic lowfrequency impulse pattern. AChE mRNA levels were determined by quantitative RT-PCR and normalized to 185 rRNA levels in the following samples: control SOL, normal soleus muscles of nontreated rats; tacrolimus SOL, soleus muscles in the tacrolimus-treated rats; stim SOL, tonically stimulated soleus muscles in nontreated rats; stim + tacrolimus SOL, tonically stimulated soleus muscles in the tacrolimus-treated rats. ${ }^{*}$ indicates statistically significantly different $(p<0.001)$ from control SOL; ${ }^{* *}$ indicates statistically significantly different $(p<0.01)$ from stim SOL.

scripts also in the muscles, electrically stimulated by the tonic low-frequency impulse pattern. In the soleus muscles (Fig. 4), the average level of AChE mRNA, determined by quantitative RTPCR after $8 \mathrm{~d}$ of tonic electrical stimulation, showed a tendency to decrease but just failed to reach statistical significance $(p=0.08)$. Tacrolimus treatment, however, significantly $(p<0.01)$ increased the average levels of AChE transcripts also in the electrically stimulated soleus muscles by approximately the same factor as in the nonstimulated control muscles.

In the next group of experiments, the specificity of tacrolimus action via calcineurin inhibition was checked. Rats were treated with CsA, another inhibitor of calcineurin activity. AChE expression in the isolated extrajunctional muscle regions was studied to ensure that the observed changes are not attributable to changes in the neuromuscular junctions. Because the extrajunctional regions cannot be reliably isolated in the fast EDL muscle, another fast muscle, the STM, was used for comparison. Quantitative RT-PCR analysis demonstrated that the AChE transcript levels in the extrajunctional regions of the soleus muscles of the rats, treated for $8 \mathrm{~d}$ by CsA, increased approximately twofold $(p<$ 0.01 ) and reached $\sim 45 \%$ of those in the extrajunctional region of the fast STM muscle (Fig. 5). As a negative control, we examined 


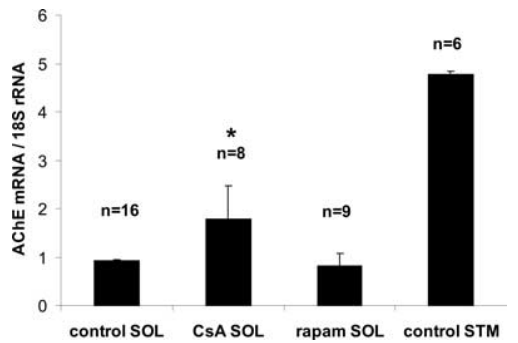

Figure 5. The effect of treatment by cyclosporin A and rapamycin on the levels of AChE in the extrajunctional regions of the slow soleus muscles; the AChE mRNA levels in the extrajunctional regions of normal fast sternomastoid muscles are shown for comparison. AChE mRNA levels were determined by quantitative RT-PCR and normalized to $18 \mathrm{~S}$ rRNA level in the following samples: control SOL, normal soleus muscles of nontreated rats; CSA SOL, the soleus muscles in the cyclosporin A-treated rats; rapa SOL, the soleus muscles in the rapamycin-treated rats; control STM, the extrajunctional region of the normal sternomastoid muscles in non-treated rats. ${ }^{*}$ indicates statistically significantly different $(p<0.01)$ from control SOL.

also a possible effect on the levels of AChE mRNA in the soleus muscle of rapamycin, which binds to the same immunophilin [FKBP12 (FK506 binding protein)] as tacrolimus but does not inhibit calcineurin. In contrast to tacrolimus and CsA, its application to rats for $8 \mathrm{~d}$ did not affect the AChE transcript levels in the soleus muscle to any significant extent $(p>0.05)$ (Fig. 5).

In contrast to the slow soleus muscles, tacrolimus treatment did not significantly change the levels of AChE transcripts in the fast EDL muscles (Fig. 6A). If the EDL muscles were electrically stimulated for $8 \mathrm{~d}$ by a chronic low-frequency impulse pattern, the average level of AChE mRNA in the stimulated muscles decreased to $\sim 40 \%$ of the control levels $(p<0.01)$. This effect, however, could be prevented by tacrolimus application: tonic electrical stimulation did not significantly decrease the average AChE transcript level in the EDL muscles of the tacrolimustreated animals compared with the control EDL muscles $(p>$ 0.05), and the AChE mRNA levels in the stimulated muscles of the treated animals remained significantly higher $(p<0.05)$ than in the stimulated muscles of the non-tacrolimus-treated animals (Fig. 6A).

AChE mRNA levels in the denervated soleus and EDL muscles of the animals treated by calcineurin inhibitors

The treatment by calcineurin inhibitors was combined with muscle denervation to examine the influence of calcineurin inhibition on muscle AChE expression in the absence of innervation. AChE mRNA levels in the extrajunctional regions of the control and denervated soleus muscles were analyzed by quantitative RTPCR (Fig. 6B). A statistically significant decrease of AChE mRNA levels to $\sim 30 \%$ of the control level was observed in the $8 \mathrm{~d}$ denervated soleus muscles compared with the controls $(p<0.001)$. In sharp contrast to the nondenervated muscles, the average level of the AChE mRNA in the denervated soleus muscles of the animals treated by tacrolimus was not statistically significantly higher than that in the denervated soleus muscles of the rats without tacrolimus treatment $(p>0.05)$. Similarly, CsA treatment of the rats also did not increase the levels of AChE mRNA in the denervated soleus muscles to any significant extent (figure not shown). Denervation, therefore, prevented the enhancing effect of calcineurin inhibition on the AChE mRNA levels in the soleus muscles. In the EDL muscles, AChE mRNA levels decreased greatly during $8 \mathrm{~d}$ after denervation and remained low $(<10 \%$ of the control value) also after calcineurin inhibition by tacrolimus,
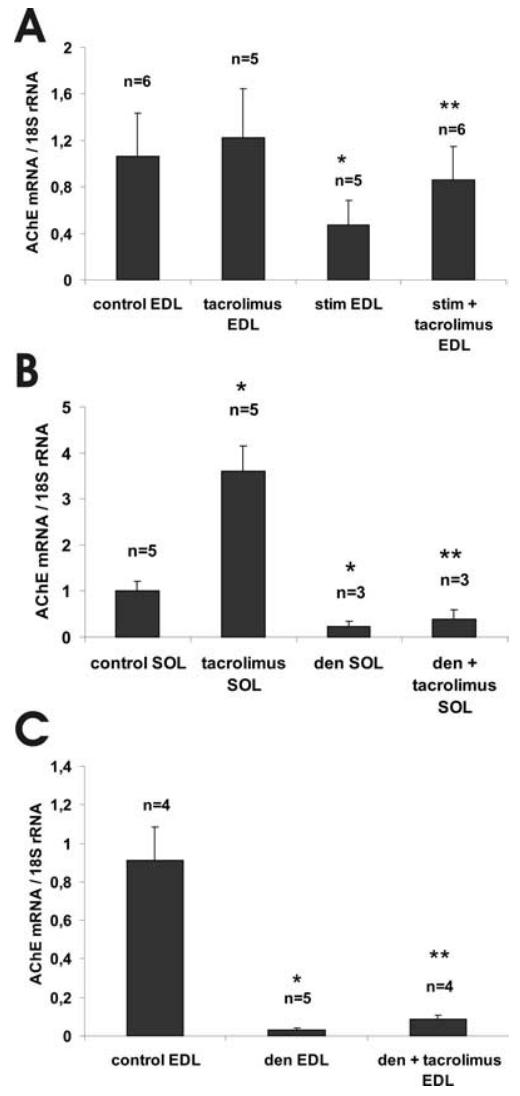

Figure 6. The effect of treatment by tacrolimus (FK506) on the levels of AChE mRNA in the control EDL muscles, the EDL muscles, electrically stimulated by the tonic low-frequency impulse pattern, and in the denervated soleus and EDL muscles. AChE mRNA levels were determined by quantitative RT-PCR and normalized to $18 \mathrm{~S}$ rRNA level in the following samples. $\boldsymbol{A}$ : control EDL, normal EDL muscles of nontreated rats; tacrolimus EDL, EDL muscles in the tacrolimus-treated rats; stim EDL, tonically stimulated EDL muscles in nontreated rats; stim + tacrolimus EDL, tonically stimulated EDL muscles in the tacrolimus-treated rats. * indicates statistically significantly different $\left(p=0.01\right.$ ) from control EDL; ${ }^{* *}$ indicates statistically significantly different $(p<0.05)$ from stim EDL but not from control EDL $(p>0.05)$. $\boldsymbol{B}$ : control SOL, normal soleus muscles of nontreated rats; tacrolimus SOL, soleus muscles in the tacrolimustreated rats; den SOL, denervated soleus muscles in nontreated rats; den + tacrolimus SOL, denervated soleus muscles in the tacrolimus-treated rats. * indicates statistically significantly different $\left(p<0.001\right.$ ) from control SOL; ${ }^{* *}$ indicates statistically significantly different ( $p<$ $0.01)$ from control SOL but not from den SOL $(p<0.05)$. c: control EDL, normal EDL muscles of nontreated rats; den EDL, denervated EDL muscles in nontreated rats; den + tacrolimus EDL, denervated EDL muscles in the tacrolimus-treated rats. * indicates statistically significantly different $(p<0.001)$ from control EDL; ${ }^{* *}$ indicates statistically significantly different from control EDL $(p<0.001)$ and also from den $\operatorname{EDL}(p=0.01)$.

despite a very small, although statistically significant, increase of the AChE transcript levels $(p=0.01)$ (Fig. 6C).

\section{Discussion}

\section{Activation pattern determines AChE mRNA levels in} muscle fibers

The present study confirmed that the levels of AChE mRNA in the rat slow soleus muscles are only $\sim 20-30 \%$ of those in the fast EDL muscles. We hypothesized that this difference was predominantly attributable to different nerve-induced patterns of fiber activation in slow (tonic, low frequency, high number of impulses per day) and fast muscles (phasic, high frequency, low number of impulses per day). Accordingly, AChE mRNA levels in low-frequency stimulated fast rat muscles decreased rapidly and became comparable with those in slow muscles (Sketelj et al., 1998). The present experiments with phasic electrical stimulation 
of muscles fully corroborated the above hypothesis. If denervated soleus muscles were electrically stimulated by a phasic highfrequency impulse pattern, the levels of muscle AChE mRNA increased and reached those in the fast EDL muscles. This increase depended on the pattern of activation: after chronic lowfrequency electrical stimulation, the AChE mRNA levels did not differ from those in the normal soleus muscles. In addition, phasic electrical stimulation of the denervated fast EDL muscles essentially prevented the huge denervation-induced decrease of AChE mRNA levels, demonstrating that high extrajunctional expression of $\mathrm{AChE}$ in fast muscles does not depend on some diffusible factors released from their motor axons. These data demonstrate that muscle activation pattern is the predominant regulator of extrajunctional AChE expression in rat muscle fibers.

Indirect phasic electrical stimulation of muscles via their motor axons imposed the phasic pattern of electrical stimulation (a short high-frequency burst each $15 \mathrm{~min}$ ) on the natural pattern of muscle activation by the nerve. Electromyographic analysis verified that the motor axons and neuromuscular junctions of the soleus muscle were able to transmit the applied short trains of high-frequency impulses to muscle fibers without failure. If this phasic electrical stimulation were applied via the nerve of an innervated soleus muscle, which maintained its natural nerveinduced tonic activation, muscle AChE mRNA levels did not increase. Therefore, occasional bursts of high-frequency activation of muscle fibers are not able to overcome the suppressive effect on AChE expression of normal tonic activation with a high number of activations per day of the soleus muscle. Second, if the EDL muscles were indirectly electrically stimulated via their motor axons with the same high-frequency phasic impulse pattern, thereby increasing the total number of high-frequency activations of the EDL muscle, AChE mRNA levels in the stimulated muscles did not further increase but decreased to $\sim 70 \%$ of the control levels. These data together indicate that a low total number of impulses per day probably are prerequisite to induce very high AChE expression in rat muscles.

\section{Calcineurin signaling pathway and muscle $\mathrm{AChE}$ regulation}

We hypothesized that the activated calcineurin signaling pathway is responsible for low AChE mRNA levels in slow muscles compared with those in the fast EDL. Tacrolimus binds to one of immunophilin proteins (FKBP12), and this complex inhibits calcineurin phosphatase activity (Liu et al., 1991). Treating rats with tacrolimus increased the extrajunctional levels of AChE transcripts in the slow soleus muscles to reach those in the fast EDL muscles and corroborated our hypothesis. Tacrolimus also increased the levels of AChE transcripts in tonically electrically stimulated soleus muscles, although to a somewhat lower level than in nonstimulated muscles, probably because a higher number of impulses per day in stimulated muscles resulted in somewhat enhanced calcineurin activity despite inhibition. Tacrolimus likewise prevented AChE mRNA decrease in the fast EDL muscles, electrically stimulated by a tonic low-frequency impulse pattern. All of this indicates that the effect of tacrolimus on AChE mRNA level is muscle cell autonomous and is not attributable to putative changes in motoneuron firing pattern, which could have occurred as a result of inhibition of calcineurin in motoneurons.

Control experiments verified the specificity of tacrolimus action. CsA, another inhibitor of calcineurin that binds to a different immunophilin (cyclophilin) from tacrolimus (Liu et al., 1991), also significantly increased the levels of AChE mRNA in the soleus muscles. Conversely, rapamycin binds to the same immunophilin (FKBP12) as tacrolimus. However, this complex does not inhibit calcineurin but blocks the phosphoinositide 3-kinase-phosphokinase B signaling pathway (Abraham, 1998; Raught et al., 2001). Accordingly, rapamycin, applied in the same dose that blocked muscle hypertrophy induced by the activation of the above pathway (Pallafacchina et al., 2002), did not significantly change the levels of AChE mRNA in the soleus muscles. Physiologically, FKBP12 in muscle fibers stabilizes the calcium release channel in the sarcoplasmic reticulum. Tacrolimus or rapamycin binding to $\mathrm{FKBP} 12$ results in loss of cooperativity between the channel subunits (Brillantes et al., 1994; Ondrias et al., 1998). Contrasting effects of tacrolimus and rapamycin on AChE expression in the soleus muscles argue against the possibility that the effect of tacrolimus in this respect is attributable to calcium release channel destabilization. A general nonspecific stimulatory effect on AChE expression in muscles is also unlikely because, in contrast to the soleus, tacrolimus did not increase AChE expression in the fast EDL muscles or in denervated muscles, in which calcineurin is inactive (Serrano et al., 2001). This was not attributable to different susceptibility to calcineurin inhibition in fast and slow muscles, because calcineurin is even more susceptible to inhibition in fast than in slow muscles (Mitchell et al., 2002). These results together strongly support the conclusion that tacrolimus increased AChE mRNA levels in slow muscles by calcineurin inhibition and not by acting on some other possible target in muscle fibers. Therefore, activated calcineurin signaling pathway decreases the levels of AChE transcripts in the extrajunctional muscle regions. Calcineurin is activated by sustained high average cytoplasmic concentration of $\mathrm{Ca}^{2+}$ typical for tonic muscle activation and is insensitive to transient, high-amplitude oscillations of $\mathrm{Ca}^{2+}$ produced by phasic high-frequency activations (Timmerman et al., 1996; Dolmetsch et al., 1997). This explains the differences in AChE expression between slow and fast muscle fibers and why a low number of muscle activations are prerequisite for high AChE transcript levels or why occasional high-frequency bursts of activity cannot overcome the suppressive effect of ongoing tonic muscle activation.

Reduction of AChE transcript levels in muscle fibers caused by the activated calcineurin pathway can be attributable to either repressed transcription of the AChE gene or enhanced transcript degradation rate. Activation of calcineurin phosphatase activity dephosphorylates cytoplasmic nuclear factor of activated T-cells (NFAT) transcription factors and translocates them into the nucleus in which they bind to the NFAT consensus sequence GGAAA in gene promoters (Rao et al., 1997). In adult rat muscles, NFATc1 stimulates slow myosin heavy chain (MyHC 1) promoter activity and inhibits the fast $\mathrm{MyHC}-2 \mathrm{~B}$ promoter $\mathrm{Mc}-$ Cullagh et al., 2004). AChE gene might be in this respect similar to the fast $\mathrm{MyHC}-2 \mathrm{~B}$. Mouse AChE promoter region contains three consensus binding sites for NFAT: one of them is conserved in mouse, rat, and human. However, the constitutively active NFATc1, instead of inhibiting, moderately stimulated the activity of the AChE gene promoter in primary rat muscle culture (Cohen and Randall, 2004). Conversely, in line with our results in adult muscles, CsA-induced inhibition of calcineurin increased the levels of AChE mRNA in cultured C2C12 myotubes, but this was primarily attributable to increased AChE mRNA stability and not to enhanced transcription (Luo et al., 1999). The transcription rates of AChE gene in adult fast and slow rat muscles are not significantly different, suggesting that changes in mRNA stability are primarily responsible for different $\mathrm{AChE}$ transcript levels in muscles (Boudreau-Lariviere et al., 2000a). The $3^{\prime}$-untranslated 
region of AChE mRNA contains an adenosine-uracil-rich element, which can change mRNA stability by binding different mRNA stabilizing or destabilizing proteins (Deschenes-Furry et al., 2003, 2005). The suppressive effect of activated calcineurin on AChE transcript levels in mature muscle fibers may therefore be posttranscriptional, i.e., mediated by changes in expression of mRNA stabilizing or destabilizing factors, which would destabilize AChE mRNA.

Denervation heavily reduced AChE mRNA levels in EDL and soleus muscles (Črešnar et al., 1994; Michel et al., 1994). Calcineurin inhibition by tacrolimus did not increase these levels to any important extent, excluding a possible role of calcineurin in this phenomenon. Denervation probably results in very low sarcoplasmic $\mathrm{Ca}^{2+}$ levels and inactive calcineurin. Seemingly, if the number of activations decreases under some threshold, then a positive regulatory mechanism, which allows for the highest AChE mRNA levels in rarely activated fast muscle fibers, is overwhelmed by increased rate of AChE mRNA degradation, typical for denervation (Grubič et al., 1999; Boudreau-Lariviere et al., 2000b), which is induced by some calcineurin-independent mechanism.

Regulation of AChE in neuromuscular junctions was not addressed in this work. However, chronic low-frequency tonic stimulation of the fast EDL muscles decreased junctional AChE activity to the low levels observed in the junctions of the slow soleus muscle (Sketelj et al., 1998). Therefore, activated calcineurin signaling mechanism might also downregulate junctional AChE expression, acting in parallel to other mechanisms regulating junctional AChE expression (Chan et al., 1999; Rossi et al., 2003; Tung et al., 2004; Cohen and Randall, 2006). The same was demonstrated for utrophin gene expression at the neuromuscular junction (Angus et al., 2005).

\section{References}

Abraham RT (1998) Mammalian target of rapamycin: immunosuppressive drugs uncover a novel pathway of cytokine receptor signaling. Curr Opin Immunol 10:330-336.

Angus LM, Chakkalakal JV, Mejat A, Eibl JK, Belanger G, Megeney LA, Chin ER, Schaeffer L, Michel RN, Jasmin BJ (2005) Calcineurin-NFAT signaling, together with GABP and peroxisome PGC-1alpha, drives utrophin gene expression at the neuromuscular junction. Am J Physiol Cell Physiol 289:C908-C917.

Ausoni S, Gorza L, Schiaffino S, Gundersen K, Lømo T (1990) Expression of myosin heavy chain isoforms in stimulated fast and slow rat muscles. J Neurosci 10:153-160.

Boudreau-Lariviere C, Chan RY, Wu J, Jasmin BJ (2000a) Molecular mechanisms underlying the activity-linked alterations in acetylcholinesterase mRNAs in developing versus adult rat skeletal muscles. J Neurochem 74:2250-2258.

Boudreau-Lariviere C, Parry DJ, Jasmin BJ (2000b) Myotubes originating from single fast and slow satellite cells display similar patterns of AChE expression. Am J Physiol Regul Integr Comp Physiol 278:R140-R148.

Brillantes AB, Ondrias K, Scott A, Kobrinsky E, Ondriasova E, Moschella MC, Jayaraman T, Landers M, Ehrlich BE, Marks AR (1994) Stabilization of calcium release channel (ryanodine receptor) function by FK506-binding protein. Cell 77:513-523.

Calvo S, Venepally P, Cheng J, Buonanno A (1999) Fiber-type-specific transcription of the troponin I slow gene is regulated by multiple elements. Mol Cell Biol 19:515-525.

Chan RYY, Boudreau-Lariviere C, Angus LM, Mankal FA, Jasmin BJ (1999) An intronic enhancer containing an N-box motif is required for synapseand tissue-specific expression of the acetylcholinesterase gene in skeletal muscle fibers. Proc Natl Acad Sci USA 96:4627-4632.

Cohen TV, Randall WR (2004) NFATcl activates the acetylcholinesterase promoter in rat muscle. J Neurochem 90:1059-1067.

Cohen TV, Randall WR (2006) The regulation of acetycholinesterase by ciselements within intron I in cultured contracting myotubes. J Neurochem 98:723-734.
Črešnar B, Črne-Finderle N, Breskvar K, Sketelj J (1994) Neural regulation of muscle acetylcholinesterase is exerted on the level of its mRNA. J Neurosci Res 38:294-299.

Delling U, Tureckova J, Lim HW, De Windt LJ, Rotwein P, Molkentin JD (2000) A calcineurin-NFATc3-dependent pathway regulates skeletal muscle differentiation and slow myosin heavy-chain expression. Mol Cell Biol 20:6600-6611.

Deschenes-Furry J, Belanger G, Perrone-Bizzozro N, Jasmin BJ (2003) Posttranscriptional regulation of acetycholinesterase mRNAs in nerve growth factor-treated PC12 cells by the RNA-binding protein HuD. J Biol Chem 278:5710-5717.

Deschenes-Furry J, Belanger G, Mwanjeve J, Lunde JA, Parks RJ, PerroneBizzozero N, Jasmin BJ (2005) The RNA-binding protein HuR binds to acetylcholinesterase transcripts and regulates their expression in differentiating skeletal muscle cells. J Biol Chem 280:25361-25368.

Dettbarn WD (1981) A distinct difference between slow and fast muscle in acetylcholinesterase recovery after reinnervation in the rat. Exp Neurol 74:33-50.

Dolmetsch RE, Lewis RS, Goodnow CC, Healy JI (1997) Differential activation of transcription factors induced by $\mathrm{Ca}^{2+}$ response amplitude and duration. Nature 386:855-858.

Grubič Z, Zajc-Kreft K, Brank M, Marš T, Komel R, Miranda AF (1999) Control levels of acetylcholinesterase expression in the mammalian skeletal muscle. Chem Biol Interact 119-120:309-319.

Hall ZW (1973) Multiple forms of acetylcholinesterase and their distribution in endplate and non-endplate regions of rat diaphragm muscle. J Neurobiol 4:343-361.

Hennig R, Lømo T (1985) Firing patterns of motor units in normal rats. Nature 314:164-166.

Hughes SM, Koishi K, Rudnicki M, Maggs AM (1997) MyoD protein is differentially accumulated in fast and slow skeletal muscle fibers and required for normal fiber type balance in rodents. Mech Dev 61:151-163.

Liu J, Farmer Jr JD, Lane WS, Friedman J, Weissman I, Schreiber SL (1991) Calcineurin is a common target of cyclophilin-cyclosporin A and FKBPFK506 complexes. Cell 66:807-815.

Lømo T, Westgaard RH, Dahl HA (1974) Contractile properties of muscle: control by pattern of muscle activity in the rat. Proc R Soc Lond B Biol Sci 187:99-103.

Luo ZD, Wang Y, Werlen G, Camp S, Chien KR, Taylor P (1999) Calcineurin enhances acetylcholinesterase mRNA stability during C2-C12 muscle cell differentiation. Mol Pharmacol 56:886-894.

McCullagh KJA, Calabria E, Pallafacchina G, Ciciliot S, Serrano AL, Argentini C, Kalhovde JM, Lomo T, Schiaffino S (2004) NFAT is a nerve activity sensor in skeletal muscle and controls activity-dependent myosin switching. Proc Natl Acad Sci USA 101:10590-10595.

Michel RN, Vu CQ, Tetzlaff W, Jasmin BJ (1994) Neural regulation of acetylcholinesterase mRNAs at mammalian neuromuscular synapses. J Cell Biol 127:1061-1069.

Mitchell PO, Mills ST, Pavlath GK (2002) Calcineurin differentially regulates maintenance and growth of phenotypically distinct muscles. Am J Physiol Cell Physiol 282:C984-C992.

Navarrete R, Vrbova G (1983) Changes of activity patterns in slow and fast muscles during postnatal development. Dev Brain Res 8:11-19.

Olson EN, Williams RS (2000) Calcineurin signaling and muscle remodeling. Cell 101:689-692.

Ondrias K, Marx SO, Gaburjakova M, Marks AR (1998) FKBP12 modulates gating of the ryanodine receptor/calcium release channel. Ann NY Acad Sci 853:149-156.

Pallafacchina G, Calabria E, Serrano AL, Kalhovde JM, Schiaffino S (2002) A protein kinase B-dependent and rapamycin-sensitive pathway controls skeletal muscle growth but not fiber type specification. Proc Natl Acad Sci USA 99:9213-9218.

Pregelj P, Črne-Finderle N, Sketelj J (2003) Effect of thyroid hormones on acetylcholinesterase mRNA levels in the slow soleus and fast extensor digitorum longus muscles of the rat. Neuroscience 116:657-667.

Rao A, Luo C, Hogan PG (1997) Transcription factors of the NFAT family: regulation and function. Annu Rev Immunol 15:115-202.

Raught B, Gingras AC, Sonenberg N (2001) The target of rapamycin (TOR) proteins. Proc Natl Acad Sci USA 98:7037-7044.

Ribarič S, Rozman J, Sketelj J (2000) Modification of skeletal muscle AChE expression by a novel method of stimulus application to the peripheral nerve. Pflügers Arch 439:R217-R219. 
Rossi SG, Dickerson IM, Rotundo RL (2003) Localization of the calcitonin gene-related peptide receptor complex at the vertebrate neuromuscular junction and its role in regulating acetycholinesterase expression. J Biol Chem 278:24994-25000.

Serrano AL, Murgia M, Pallafacchina G, Calabria E, Coniglio P, L $\phi$ mo T, Schiaffino S (2001) Calcineurin controls nerve activity-dependent specification of slow skeletal muscle fibers but not muscle growth. Proc Natl Acad Sci USA 98:13108-13113.

Simoneau JA, Pette D (1988) Species-specific effects of chronic nerve stimulation upon tibialis anterior muscle in mouse, rat, guinea pig, and rabbit. Pflügers Arch 412:86-92.

Sketelj J (1994) Neural regulation of acetylcholinesterase in skeletal muscles. Basic Appl Myol 4:281-291.

Sketelj J, Brzin M (1985) Asymmetric molecular forms of acetylcholinesterase in mammalian skeletal muscles. J Neurosci Res 14:95-103.

Sketelj J, Črne-Finderle N, Ribarič S, Brzin M (1991) Interactions between intrinsic regulation and neural modulation of acetylcholinesterase in fast and slow skeletal muscles. Cell Mol Neurobiol 11:35-54.

Sketelj J, Leisner E, Gohlsch B, Škorjanc D, Pette D (1997) Specific impulse patterns regulate acetylcholinesterase activity in skeletal muscles of rats and rabbits. J Neurosci Res 47:49-57.

Sketelj J, Črne-Finderle N, Štrukelj B, Trontelj JV, Pette D (1998) Acetylcho- linesterase mRNA level and synaptic activity in rat muscles depend on nerve-induced pattern of muscle activation. J Neurosci 18:1944-1952.

Spangenburg EE, Williams JH, Roy RR, Talmadge RJ (2001) Skeletal muscle calcineurin: influence of phenotype adaptation and atrophy. Am J Physiol Regul Integr Comp Physiol 280:R1256-R1260.

Swoap SJ, Hunter RB, Stevenson EJ, Mitchell Felton H, Kansagra NV, Lang JM, Esser KA, Kandarian SC (2000) The calcineurin-NFAT pathway and muscle fiber-type gene expression. Am J Physiol Cell Physiol 279:C915-C924.

Termin A, Staron RS, Pette D (1989) Changes in myosin heavy chain isoforms during chronic low-frequency stimulation of rat fast hindlimb muscles. A single-fiber study. Eur J Biochem 186:749-754.

Timmerman LA, Clipstone NA, Ho SN, Northrop JP, Crabtree GR (1996) Rapid shuttling of NF-AT in discrimination of $\mathrm{Ca}^{2+}$ signals and immunosuppression. Nature 383:837-840.

Trontelj JV (2003) Single fiber electromyography. In: Clinical neurophysiology of disorders of muscle and neuromuscular junction, including fatigue, Handbook of clinical neurophysiology, Vol 2 (Stålberg E, ed), pp 187-211. Amsterdam: Elsevier.

Tung EK, Choi RC, Siow NL, Jiang JX, Ling KK, Simon J, Barnard EA, Tsim KW (2004) P2Y2 receptor activation regulates the expression of acetylcholinesterase and acetylcholine receptor genes at vertebrate neuromuscular junction. Mol Pharmacol 66:794-806. 